\title{
Inflammasome assembly: The wheels are turning
}

\author{
Cell Research (2015) 25:1277-1278. doi:10.1038/cr.2015.137; published online 24 November 2015
}

\begin{abstract}
Inflammasomes control host cell death and inflammation in response to sterile or infectious stimuli. Two recent reports published in Science reveal the structural basis for the assembly of NAIP-NLRC4 inflammasomes.
\end{abstract}

The innate immune response relies on germline-encoded pattern-recognition receptors that monitor the extracellular and intracellular compartments of host cells for the presence of pathogens. Detection of microbe-derived ligands or endogenous danger signals in the host cell cytosol can result in the assembly of large multi-protein complexes termed inflammasomes, which drive the activation of caspase-1, a cysteine protease. Once activated, caspase-1 initiates a pro-inflammatory cell death program called pyroptosis, and promotes the maturation and secretion of cytokines like interleukin (IL)-1 $\beta$ and -18 . While inflammasomes play a protective role against microbial infections, uncontrolled inflammasome activation is implicated in chronic inflammatory disorders such as atherosclerosis, gout and diabetes.

NLRC4, a member of the nucleotidebinding and oligomerization domain (NOD)-like receptor (NLR) family, assembles an inflammasome in response to bacterial pathogens. Although often referred to as a receptor, NLRC4 does not directly bind microbe-derived ligands, but employs so-called NAIP proteins (NLR family, apoptosis inhibitory protein) as upstream sensors for different ligands $[1,2]$. In mice, NAIP5/6 were shown to recognize bacterial flagellin, while the inner rod and the needle subunit of the Salmonella typhimurium Type 3 Secretion System is recognized by NAIP2 and NAIP1, respectively. Previous work showed that ligand binding induces the interaction of NAIPs with NLRC4 and the formation of disk-shaped oligomeric complexes [3], but the underlying mechanism was unknown.

Zhang et al. [4] and $\mathrm{Hu}$ et al. [5] have now used Cryo-electron microcopy (Cryo-EM) to examine the structure of the PrgJ-NAIP2-NLRC4 complex and its assembly mechanism. Both studies found that the complex has a wheel- or disk-like architecture, with 10-12 spokes corresponding to individual protomers. While it was not possible to distinguish NAIP2 and NLRC4 protomers within the complex, probably due to their conserved domain organization, analysis of unfinished NAIP2-NLRC4 disks indicated that the protomer at one end is different from the remaining subunits [5]. Nanogold labeling demonstrated that only one NAIP2 is found per complex, thus supporting a model in which the assembly of the entire NAIP2NLRC4 inflammasome starts with the ligand-induced activation of one single NAIP molecule. As the authors point out that this differs strikingly from the assembly of the heptameric apoptosome, a caspase- 9 activating platform, which requires ligand-mediated activation of every Apaf-1 protomer of the complex $[4,5]$.

How does activated NAIP2 induce the progressive oligomerization of NLRC4? Structural comparison of the atomic model of the NAIP2-NLRC4 complex and the previously determined crystal structure of inactive monomeric NLRC4 [6] revealed that a striking $\sim 90^{\circ}$ hinge rotation accompanies NLRC4 activation. This conformational change results in the formation of a new oligomerization surface (referred to as catalytic or nucleating surface) that interacts with the next protomer in the wheel-like structure and thus facilitates progressive oligomerization (Figure 1). A matching receptor surface is preformed in the inactive NLRC4 molecule, and mediates the recruitment of NLRC4 into the growing complex. Intriguingly, missense mutations in NLRC4 that are associated with autoinflammatory conditions map to this important hinge region [7-9]. Whether such mutations induce the formation of NLRC4 disks in the absence of activated NAIPs remains to be shown, but the new studies provide the structural basis for understanding these gain-of-function mutations.

Both studies also found that NAIP2 itself was precluded from self-oligomerization and further recruitment into the complex. Structural analysis showed that all NAIP family members feature a catalytic or nucleating surface, which interacts with the receptor surface of inactive NLRC4 and induces the activating conformational changes that mediate progressive oligomerization. However, in contrast to NLRC4, NAIPs lack a receptor surface matching the nucleating surface of their own or of NLRC4. Thus, NAIPs only act as initiators of inflammasome disk formation and only one single NAIP member can be found to be incorporated into a NAIP-NLRC4 complex.

Revealing the mechanism of NAIPNLRC4 inflammasome formation is a major breakthrough, but many mysteries remain. An important next step is 


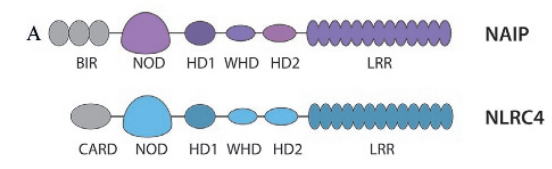

$$
\text { B }
$$

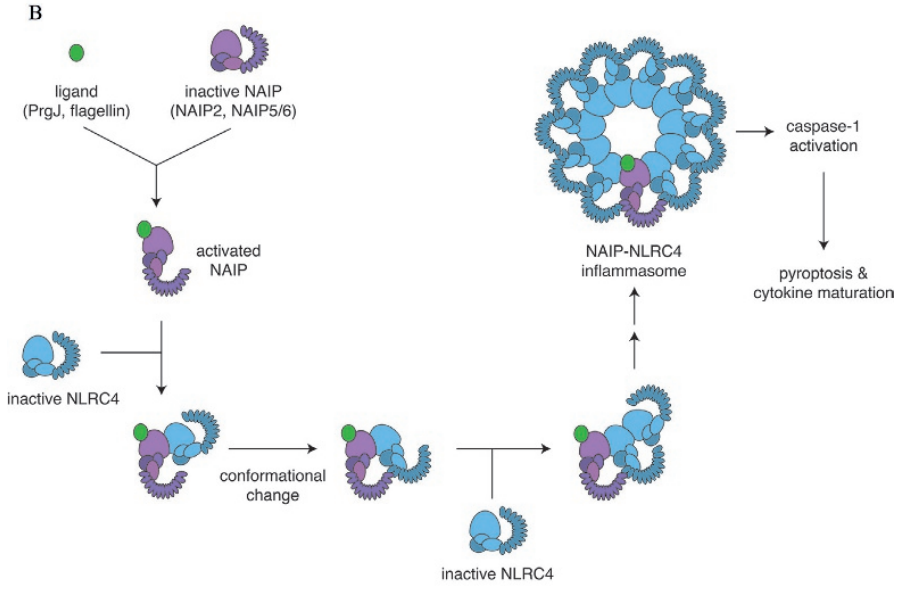

Figure 1 (A) Domain organization of NAIPs and NLRC4 in mice. BIR, Baculovirus Inhibitor of apoptosis protein Repeat; NOD, nucleotide-binding and oligomerization domain; HD1, helical domain 1; WHD, winged-helical domain; HD2, helical domain 2; LRR, leucine-rich repeats; CARD, caspase-recruitment domain. (B) Assembly mechanism of NAIP-NLRC4 inflammasomes. Binding of their specific ligand activates mouse NAIP proteins. The activated NAIP interacts with an inactive NLRC4 molecule and induces a conformational change that activates NLRC4, resulting in the generation of a new catalytic or nucleating surface. Active NLRC4 molecules recruit and activate NLRC4 molecules in a domino-like reaction. The completed NAIP-NLRC4 inflammasome is a multi-subunit disk-like structure containing 9-11 molecules of NLRC4, but only one NAIP molecule. The NLRC4CARD (not shown for simplicity reasons) initiates caspase-1 activation either through the adaptor protein ASC or directly. Active caspase-1 promotes inflammation and host defense by inducing pyroptotic cell death and cytokine maturation.

to define how the wheel-like complex is connected to the downstream signaling components. NLRC4 features a caspase-recruitment domain (CARD) that can either recruit pro-caspase-1 or ASC, a small adaptor protein featuring a CARD and a Pyrin domain (PYD), through which it oligomerizes to form a larger macromolecular complex known as "ASC speck". Zhang et al. [4] and Hu et al. [5] used CARD-deleted NLRC4 for their studies to avoid unspecific oligomerization of the CARD, therefore the orientation of the NLRC4 ${ }^{\text {CARD }}$ within the disk could not be determined. Consistent with the ability of NLRC4 to directly interact with and activate caspase-1 even in the absence of ASC, Zhang et al. [4] showed that complexes containing full-length NLRC4 induce the oligomerization of the caspase-1 $1^{\text {CARD }}$.
Yet since ASC is recruited to activated NLRC4 in wild-type cells, it will be also interesting to determine whether and how NAIP-NLRC4 complexes initiate ASC filaments, and how such a macromolecular structure looks like.

Another open question is how the ligand PrgJ induces NAIP activation and where it is located within the wheel-like structure. Previous work showed that the NOD domain of NAIPs confers ligand binding and specificity [10], yet according to the new studies the same domain also forms the nucleating surface that initiates complex assembly. Since thus far the structure of inactive or activated NAIPs has not been reported, the structural basis underlying ligand-NOD domain interaction is unknown and the resulting conformational changes remain to determined.
Finally, it needs to be investigated whether the mechanism of NAIP-NLRC4 complex formation applies to other types of inflammasomes. The PYHIN family member AIM2, which activates caspase-1 upon recognizing pathogenor host-derived DNA in the cytosol, was proposed to oligomerize along doublestranded DNA instead of forming wheellike structures. Nonetheless, other NLR family members like NLRP3 might form disk-shaped inflammasomes in analogy to the NAIP-NLRC4 complex. Indeed, gain-of-function mutations in NLRP3 that are associated with autoinflammatory disorders were shown to result in auto-oligomerization [11], but the Cryo-EM structure of such complexes has not yet been solved. Determining the structure of other inflammasomes will thus be an exiting field of research for structural biologist and might reveal important insight into the activation mechanisms of these receptors.

\section{Petr Broz ${ }^{1}$}

${ }^{1}$ Focal Area Infection Biology, Biozentrum, University of Basel, Basel, Switzerland

Correspondence: Petr Broz

E-mail: petr.broz@unibas.ch

\section{References}

1 Kofoed EM, Vance RE. Nature 2011; 477:592-595.

2 Zhao Y, Yang J, Shi J, et al. Nature 2011; 477:596-600.

3 Halff EF, Diebolder CA, Versteeg M, et al. J Biol Chem 2012; 287:38460-38472

4 Zhang L, Chen S, Ruan J, et al. Science 2015; 350:404-409

5 Hu Z, Zhou Q, Zhang C, et al. Science 2015; 350:399-404.

$6 \mathrm{Hu}$ Z, Yan C, Liu P, et al. Science 2013; 341:172-175.

7 Romberg N, Al Moussawi K, Nelson-Williams C, et al. Nat Genet 2014; 46:11351139.

8 Canna SW, de Jesus AA, Gouni S, et al. Nat Genet 2014; 46:1140-1146.

9 Kitamura A, Sasaki Y, Abe T, et al. J Exp Med 2014; 211:2385-2396.

10 Tenthorey JL, Kofoed EM, Daugherty MD, et al. Mol Cell 2014; 54:17-29.

11 Baroja-Mazo A, Martín-Sánchez F, Gomez AI, et al. Nat Immunol 2014; 15:738-748. 